\title{
Advanced Methods for Perfusion Analysis in Echocardiography
}

\author{
R. KOLÁ $\check{\mathbf{R}}^{1}$, R. JIŘÍK ${ }^{1}$, V. HARABIŠ ${ }^{1}$, M. MÉZL ${ }^{1}$, M. BARTOŠ ${ }^{1}$ \\ ${ }^{1}$ Department of Biomedical Engineering, Faculty of Electrical Engineering and Communication, \\ Brno University of Technology, Brno, Czech Republic
}

Received February 5, 2010

Accepted March 26, 2010

\begin{abstract}
Summary
It has been shown that besides positron emission tomography, single photon emission computed tomography and magnetic resonance imaging; contrast echocardiography can be used for qualitative and quantitative myocardial perfusion assessment. In this review, the properties of ultrasound contrast agents, imaging techniques and acquisition methods are shortly described and the possibilities of perfusion echocardiography are summarized. The main focus is put on the description of three perfusion models: mathematical models, physical models assuming an ideal inflow and physical models including inflow measurement.
\end{abstract}

\section{Key words}

Echocardiography • Myocardial perfusion • Ultrasound contrast agent

\section{Corresponding author}

R. Kolář, Department of Biomedical Engineering, Faculty of Electrical Engineering and Communication, Brno University of Technology Kolejní 4, Brno, 612 00, Czech Republic. E-mail: kolarr@feec.vutbr.cz

\section{Introduction}

The diagnostic possibilities of echocardiography have been extended with the development of ultrasound contrast agents (UCAs). These gas-filled microbubbles are administered into the blood stream and can be easily detected due to their nonlinear behavior (Hoff 2001). Over the last two decades the UCAs became a standard tool in different clinical and experimental applications, particularly in echocardiography. Several review papers have been already published on these topics, see e.g. (Mulvagh 2000, Lang and Mor-Avi 2006, Rakhit et al. 2007) and the reference therein.

The advantage of UCAs application has been reported to improve detection of blood-tissue interface, which enables to improve assessment of ventricular wall motion and delineation of endocardium (Hundley at al. 1998, Kornbluth et al. 2000) or some abnormalities of coronary arteries (Kaul et al. 1997). Another potential of the UCA application lies on tissue perfusion analysis. While this application field is still more experimental than clinical, first results indicate the usefulness for qualitative and quantitative perfusion assessment in simulated phantom studies (Potdevin et al. 2002, Arditi et al. 2006), in echocardiography (Wei et al. 1998, Hossack et al. 2004) and other applications like renal perfusion (Kaul et al. 2001, Forsberg et al. 2004) or cerebral perfusion (Rim et al. 2001, Eyding et al. 2002). This paper describes the principle of the perfusion methods and gives an overview of the underlying models and their applications focused on myocardial perfusion.

\section{Contrast agents and perfusion imaging techniques}

The first generation of UCAs had many disadvantages (rapid dissolving in blood and large diameter) which limited their applications predominantly to cardiac shunt detection and examination of right heart structures. Recently, UCAs have attained a prolonged persistence and smaller mean diameter, around $2 \mu \mathrm{m}$, which can be achieved using a special bubble shell (lipid, polymer etc.) and filling gas (perfluoropropane, 
perfuorocarbon etc.). The list of the second generation of UCAs may be found for example in (Mulvagh 2000). The images and results presented below were obtained with the SonoVue ${ }^{\odot}$ contrast agent. The shell of these microbubbles consists of a flexible membrane of phospholipids with a hydrophilic surface on the outside and a hydrophobic surface on the inside. The gas in SonoVue microbubbles is sulphur hexafluoride (Greis 2004).

The UCAs are intravascular contrast agents, as the vessel wall is not permeable for microbubbles. The high echogenity of the UCAs allows tracking of the contrast bubbles in the blood and hence examining of the tissue perfusion. Two techniques have been developed for perfusion analysis on the microcapilary level.

The first imaging method, called destructionreplenishment, uses continuous (or slow) UCA bolus injection. For each imaging sequence, several destructive ultrasound pulses with high ultrasound power (mechanical index MI>1) are transmitted into imaging tissue to destroy the contrast bubbles in the imaged plane. This destruction is followed by a low power imaging phase (with low MI, around 0.1) to measure the rate of UCA replenishment (Rim et al. 2001). The time-varying image intensity $I(t)$ in a specific region of interest (ROI), which corresponds to the UCA concentration, can be modeled as $I(t)=A\left(1-e^{-\beta t}\right)$, where $\beta$ represents the rate of the UCA replenishment and $A$ is the steady ROI intensity. It can be shown that $A \beta$ is proportional to the blood flow through the ROI and $A$ is proportional to the blood volume in the ROI (e.g. Rim et al. 2001).

The second imaging method uses fast UCA bolus injection followed by a flush of normal saline solution. This approach is often referred to as a bolus kinetic (Eyding et al. 2002) technique and will be described in more detail in the following sections.

\section{Data acquisition and preprocessing}

The image sequence used for perfusion analysis is recorded following the contrast agent administration. The aim is to record the UCA concentration development in time for particular myocardial ROIs. The physical quantity proportional to the UCA concentration is the backscattered ultrasound intensity (Bartoš et al. 2008), calculated as the mean intensity within the ROI. To improve the contrast between the signal backscattered from the tissue and from the UCA, second harmonic signal is utilized. This approach is based on highly nonlinear scattering of the UCA, in contrast to fairly linear scattering in the tissue. Hence, the ultrasound scanners are used in the pulse inversion, power modulation or harmonic imaging modes (Vannan 2000).

One of the major problems in perfusion analysis in echocardiography is the suppression of displacement in the successive images in the recorded sequence. This is mainly due to the movement of the patient, ultrasound probe and breathing. Alignment of displaced images (image registration) is based on searching for an optimal geometrical transformation of the sequence images with respect to one reference image from the sequence (Gardner 2004, Kolar et al. 2009).

Another important issue has been suppression of attenuation. This is important especially in echocardiography, where the intensity of reflected ultrasound waves from myocardium segments is influenced not only by the UCA concentration in it but also by the UCA concentration in the heart cavity between the ROI and the probe. Available solutions are not robust and automated enough yet (e.g. Mischi et al. 2005, Zwirn et al. 2009).

\section{Perfusion models}

During perfusion analysis a fast UCA bolus is administered into the blood stream and the image sequence is recorded. The ROI defines a specific myocardial tissue for perfusion analysis (see Fig. 1a). The fast bolus imaging method results in a perfusion curve (Fig.1b) extracted from the myocardial ROI. Processing and analysis of these perfusion curves representing the time-varying concentration lead to estimation of the parameters related to the perfusion in the ROI. Based on the model complexity, these parameters may be heuristic indices related to the perfusion state of the tissue or they can be physical quantities based on physical modeling of the perfusion process. Three quantitative parameters related to the myocardium perfusion properties on the microvascular level may be estimated:

1. Mean transit time (MTT) - average time needed for bubbles to pass through the ROI in [s].

2. Blood volume $\left(\mathrm{V}_{\mathrm{b}}\right)$ - volume of blood in the ROI per tissue weight $[\mathrm{ml} / \mathrm{g}]$.

3. Blood flow $\left(\mathrm{F}_{\mathrm{b}}\right)$ - blood flow $[\mathrm{ml} / \mathrm{min}]$ in the ROI per tissue weight $[\mathrm{ml} / \mathrm{min} / \mathrm{g}]$.

Two additional physiological values have to be known to be able to estimate these parameters: tissue density and hematocrit correction factor, etc. (Jackson 

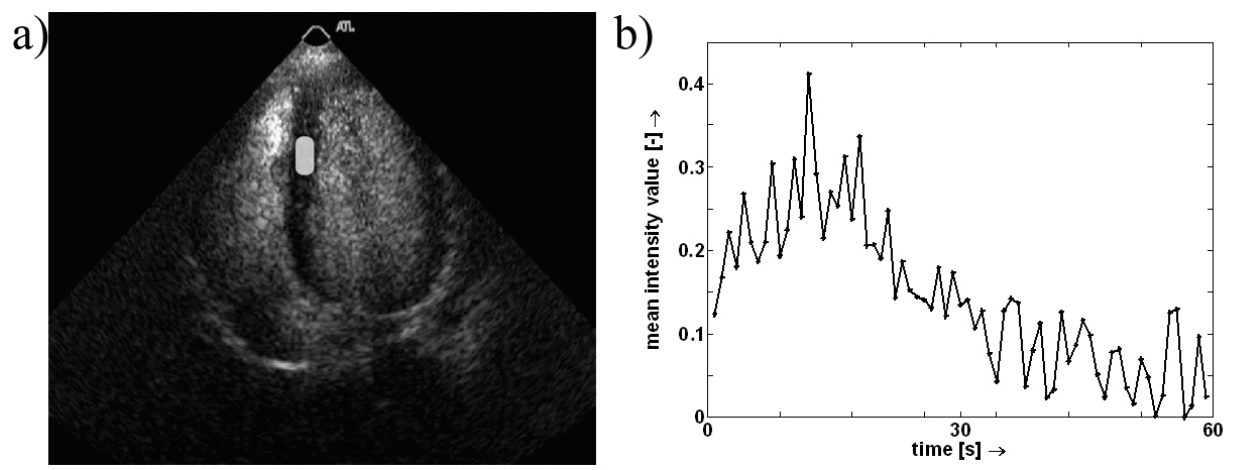

Fig. 1. a) One image from recorded sequence with ROI. b) Time varying mean ROI intensity, which corresponds to the UCA concentration $c(t)$. c) Measured intensity and fitted curves using Log-Normal and Erlang model. d) Measured intensity and fitted curves using Gamma and LDRW model.
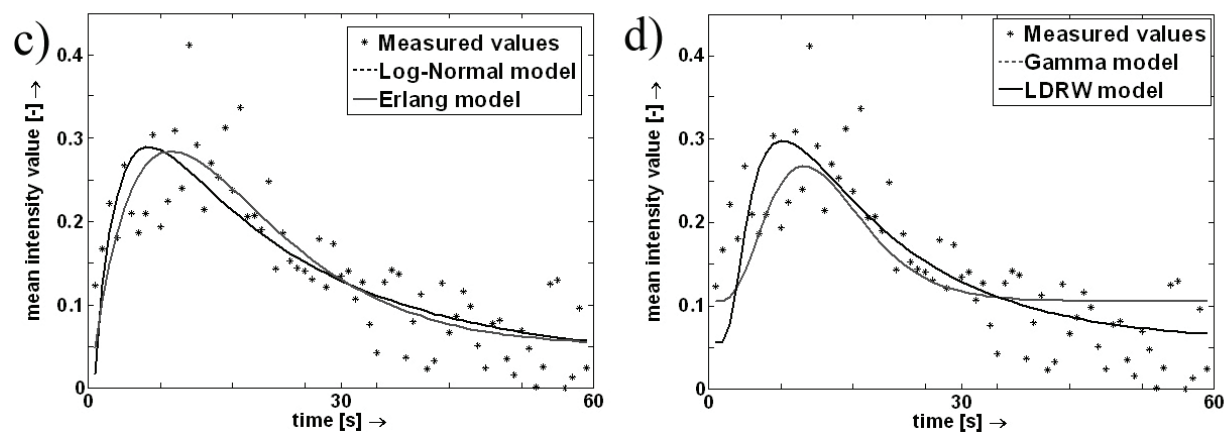

et al. 2005).

Three different approaches for qualitative or quantitative perfusion description have been reported. They will be described in the following sections. The mathematic formulas are included for comprehensiveness.

\section{Mathematical models}

These models are based on the shape similarity between the perfusion curve $c(t)$ and a specific mathematical function used for data fitting. There is no exact physical interpretation of the model parameters. The Log-Normal distribution $f_{\text {Log-Norm }}(t)$ is a favorite fitting function in dilution techniques (e.g. Rognin 2008) and is therefore convenient in perfusion applications. It is defined as:

$$
f_{\text {Log-Norm }}(t)=\frac{1}{t \sigma \sqrt{2 \pi}} e^{-\frac{(\ln (t)-\mu)^{2}}{(2 \sigma)^{2}}}
$$

where the parameters $\mu$ and $\sigma$ define the shape of this function and must be estimated to fit the shape of the measured curve (see Fig. 1c). These parameters describe indirectly the perfusion state of the analyzed myocardium.

\section{Physical models assuming an ideal inflow}

These models are based on a physical description of the tissue perfusion and describe the cardiovascular system between the bolus application and the tissue ROI. Furthermore, an ideal bolus (infinitely short injection, but in practice very fast bolus injection in comparison to MTT) is assumed at the input of the myocardial ROI. Two models which are used for perfusion modeling are adopted from dilution techniques: compartment model with an ideal input (e.g. Mischi et al. 2008) and the random walk model with an ideal input (Mischi et al. 2005).

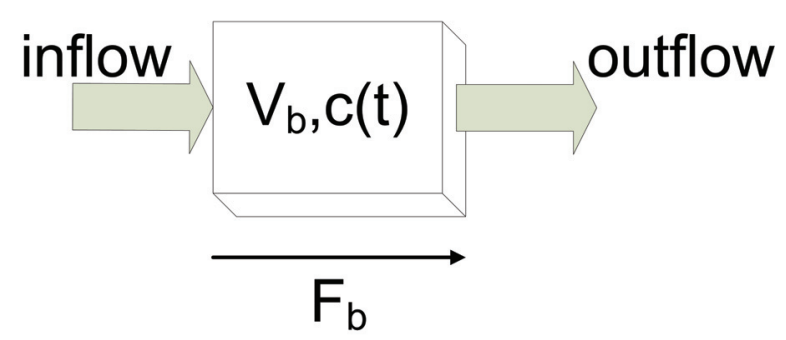

Fig. 2. Single compartment with a constant blood volume $V_{b}$, constant blood flow $F_{b}$ and a time varying UCA concentration $c(t)$.

Compartment modeling is based on a mathematical description of a small ideal mixing chamber with a unidirectional UCA washout and with a constant flow rate $F_{b}$ (Fig. 2). Each part of the cardiovascular system can be considered as one compartment, e.g. left ventricle, a part of some artery or a perfused myocardium region. The modeled UCA concentration $f_{c}(t)$ in one compartment after applying an ideal bolus can be 
mathematically described as an exponentially decreasing function:

$$
f_{c}(t)=c_{0} e^{-\frac{t}{\tau}}
$$

where $\tau$ is a time constant depending on the blood flow rate (i.e. perfusion) and the compartment volume as $\tau=\mathrm{V}_{\mathrm{b}} / \mathrm{F}_{\mathrm{b}}$ and $\mathrm{c}_{0}$ is the UCA concentration at time $\mathrm{t}=0$ (the beginning of the measurement). This simplest model of one compartment can be extended to a more complex multi-compartment model. This leads to Erlang distribution (Mischi et al. 2008):

$$
f_{\text {Erlang }}(t)=c_{0} \frac{e^{-\frac{t}{\tau}}\left(\frac{t}{\tau}\right)^{(k-1)}}{(k-1) !},
$$

where the new parameter $k$ defines the number of compartments. An example of perfusion curve fitting using this model is shown in Figure 1c.

Generalization (for non-integer $k$ ) of this model is also used and is called Gamma distribution (Mischi et al. 2008):

$$
f_{\text {Gamma }}(t)=c_{0} \frac{e^{-\frac{t}{\tau}}\left(\frac{t}{\tau}\right)^{(k-1)}}{\Gamma(k)}
$$

where $\Gamma(k)$ represents a special type of a mathematical function, called Gamma function (Rektorys 1994). An example of perfusion curve fitting using this generalized model is shown in Figure 1d.

The local density random walk (LDRW) model has been introduced by Sheppard and Savage (Sheppard and Savage 1951) and adopted for estimation of the ejection fraction using UCA by Mischi (Mischi et al. 2005). This LDRW model can be used also for perfusion analysis where it describes an ideal injection of the UCA bolus and its behavior in a straight infinitely-long tube. Due to a constant blood velocity and Brownian motion of the UCA bubbles, the time-varying concentration can be described as (Mischi et al. 2005):

$$
f_{\text {LDRW }}(t)=\frac{1}{\mu} e^{\lambda} \sqrt{\frac{\lambda \mu}{2 \pi t}} e^{-\frac{\lambda}{2}\left(\frac{t}{\mu}+\frac{\mu}{t}\right)},
$$

where $\mu$ is the mean transit time through the modeled tube and $\lambda$ determines the type of the UCA behavior in the blood stream. Both parameters are related to the ROI perfusion. An example of curve fitting using this model is shown in Figure 1d.

\section{Physical models including inflow measurement}

This approach has been extensively used in perfusion imaging using positron emission tomography (PET) (Akinboboye et al. 2001), electron beam computed tomography (EBCT) (Mohlenkamp et al. 2003) and magnetic resonance imaging (Ostergaard 2005), but so far, surprisingly, very little in ultrasonography (Quaia 2005). It is also partly based on the compartment theory. However, compared to the above described physical models, these models describe the UCA kinetics directly for the ROI. The effect of the cardiovascular system between the bolus-administration location and the ROI and the non-ideal bolus administration are included in the arterial input function (AIF). In myocardial perfusion applications the $A I F(t)$ is usually measured as the perfusion curve in the left ventricle (LV), because blood from LV (from the aorta, respectively) directly fills the coronary arteries. The change of the AIF between the measurement location and the ROI location is neglected.

Myocardial ROI can be considered either as a homogenous compartment or, more generally, as a complicated microvasculature region. The latter model is based on a concept of the tissue residue function $R(t)$ (Ostergaard 2005), which can be considered as the normalized UCA concentration curve that would be measured in the ROI in case of an ideal AIF.

Having $\operatorname{AIF}(t)$ measured in the left ventricle and $c(t)$ in the analyzed myocardium, three quantitative perfusion parameters can be estimated (blood volume, blood flow and mean transit time) from the following equations. Blood volume $V_{b}$ can be estimated directly from the measured curves $c(t)$ and $A I F(t)$ and the assumed tissue density $\rho$ :

$$
V_{b}=\frac{1}{\rho} \frac{\int c(t) d t}{\int A I F(t) d t},
$$

where the symbol $\int$ denotes the area under the respective measured curve. Blood flow $F_{b}$ can be estimated from:

$$
c(t)=\rho F_{b} R(t) * A I F(t)
$$

using appropriate mathematic operations, including deconvolution and assuming that $R(t=0)=1$ (Ostergaard 2005). The third perfusion parameter is the mean transit time described above. It is related to the blood flow and 
blood volume as (Ostergaard 2005):

$$
M T T=\frac{V_{b}}{F_{b}} .
$$

\section{Results and discussion}

Three examples related to the myocardium perfusion analysis are given in this section. The presented results are based on our experiments with GE VingMed System 5 ultrasound scanner and SonoVue contrast agent. The analyzed contrast sequences were acquired after UCA bolus administration of $2.5 \mathrm{mg}$, followed by a saline flush bolus. The recordings were triggered by ECG and only end-systolic and end-diastolic images were recorded from each cardiac cycle to decrease the probability of bubble destruction.

\section{Experiment 1}

As mentioned above, one of the main difficulties is the time dependent attenuation caused by time varying UCA concentration in the LV. Therefore, the attenuation coefficient has to be estimated before perfusion analysis in order to obtain reliable results. This can be done by employing the exponential-decay model (Zwirn et al. 2009) and rough manual LV segmentation. An example of the attenuation coefficient estimation is depicted in Figure 3a for the end-diastolic sequence. These numbers correlate with the attenuation values estimated in perfusion analysis of experimental animals (Mule et al. 2008) and in vitro phantom studies (Chen et al. 2002). The curve shape follows the increasing and decreasing UCA concentration during dilution process of the bubbles in the left ventricle. Figure $3 \mathrm{~b}$ shows how this attenuation coefficient influences the perfusion curve from defined myocardium ROI. The perfusion curve before the correction lacks the typical dilution peak, but after attenuation compensation the curve follows the expected shape.

\section{Experiment 2}

In this experiment, perfusion was examined in the apical, mid and basal segments of the myocardial septum in the end-systolic phase (Fig. 4a). Three ROIs were manually placed in the corresponding segments and perfusion curves were extracted from the recorded sequence. The analysis was based on curve-fitting using the LDRW model (Fig. 4 b, c, d), where the perfusion

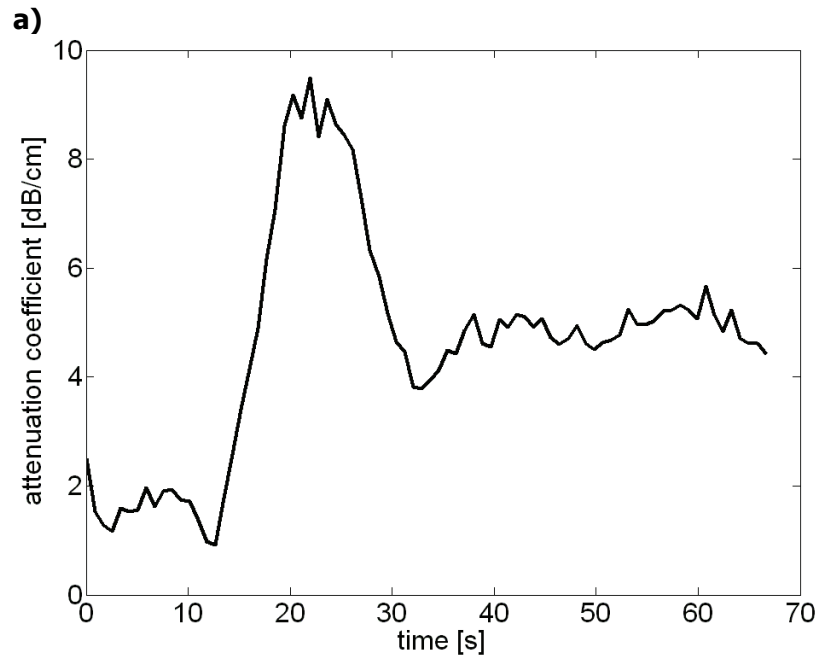

b)

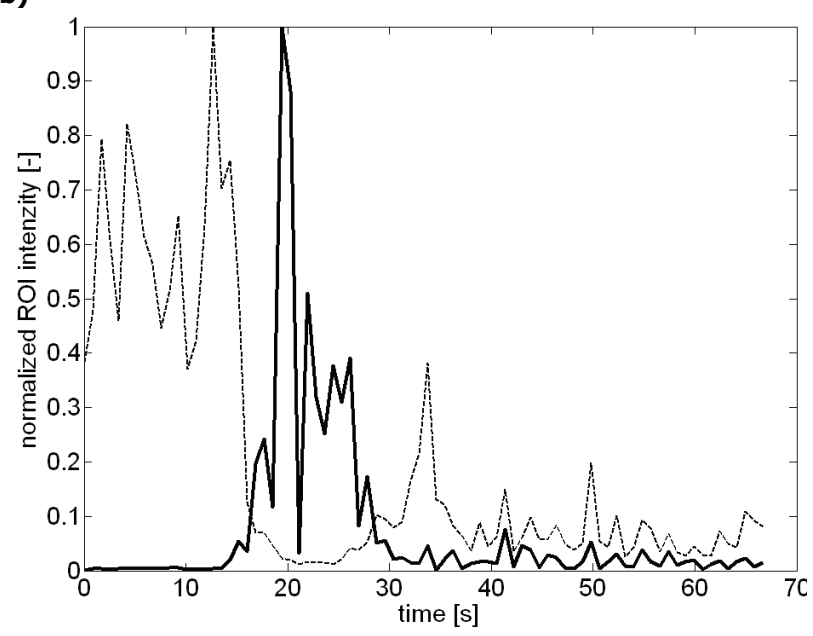

Fig. 3. a) The estimation of the time-varying attenuation coefficient. b) The perfusion curves before (dashed line) and after attenuation compensation (solid line).

parameter MTT is directly represented by model parameter $\mu$ (see equation 5). The results of this estimation are: $M T T_{\text {basal }}=6.3 \mathrm{~s}$, MTTmid $=9.6 \mathrm{~s}$ and $M T T_{\text {apical }}=10.3 \mathrm{~s}$, which correlates with findings in (Malm et al. 2005), where destruction-replenishment approach has been used.

\section{Experiment 3}

The intensity curves $A I F(t)$ (Fig. 5, left) and $c(t)$ (Fig. 5, right) were measured in the left ventricle and the mid posterior segment of the myocardium, respectively (healthy volunteer recording). Before processing, the curves were smoothed by averaging using a 7-sample window. The tissue residue function (Fig. 5, middle) was estimated by a method of parametric deconvolution assuming a compartment model of the ROI. Figure 5, right, shows the correspondence of the measured 
a)

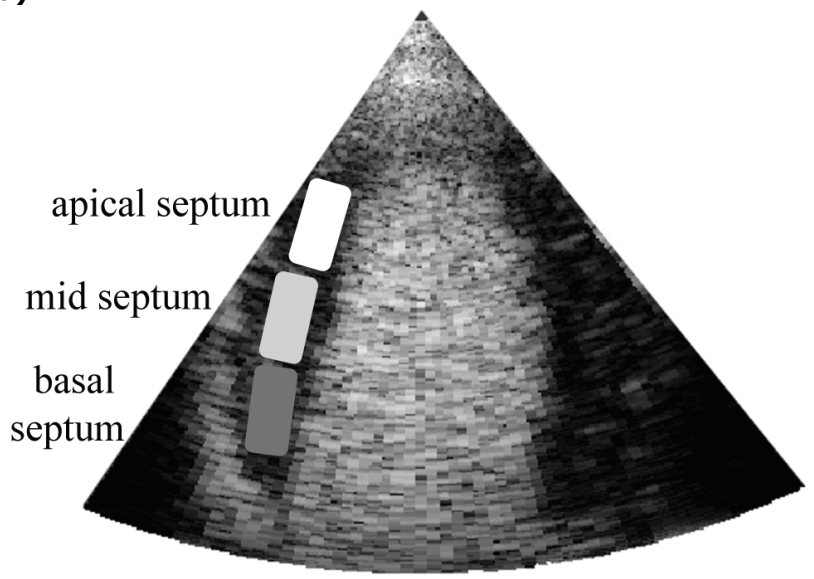

b)

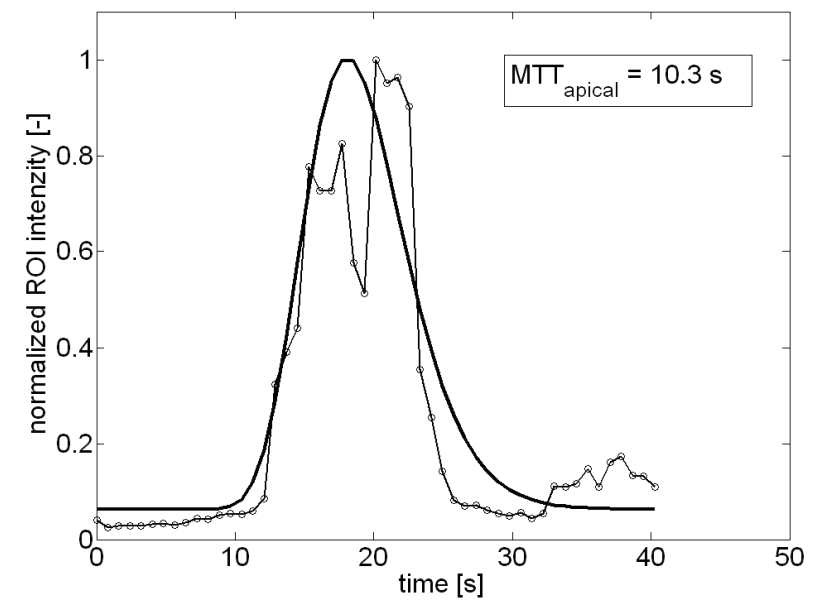

perfusion curve $c(t)$ and its approximation by the convolution of $A I F(t)$ and $R(t)$. The above perfusion parameter estimation results in $F_{b}=41 \mathrm{ml} / \mathrm{min} / 100 \mathrm{~g}, V_{b}=$ $5 \mathrm{ml} / 100 \mathrm{~g}, M T T=8 \mathrm{~s}$, which is in the range reported for myocardium using other perfusion analysis methods (e.g. Mohlenkamp et al. 2003).

\section{Conclusion}

This review has described the possibilities and problems of perfusion analysis in echocardiography. There are two main approaches: destructionreplenishment method and bolus-kinetic method. Here, the main focus has been put on the bolus kinetic approach and its mathematical description, because it is still in a research stage and might be of high diagnostic potential. The principal advantage of this method is that the bolus injection is cheaper and easier to perform than the destruction-replenishment method, where automatic injection equipment and a higher UCA dose is needed to obtain reliable results.

Dynamic contrast-enhanced echocardiography is c)

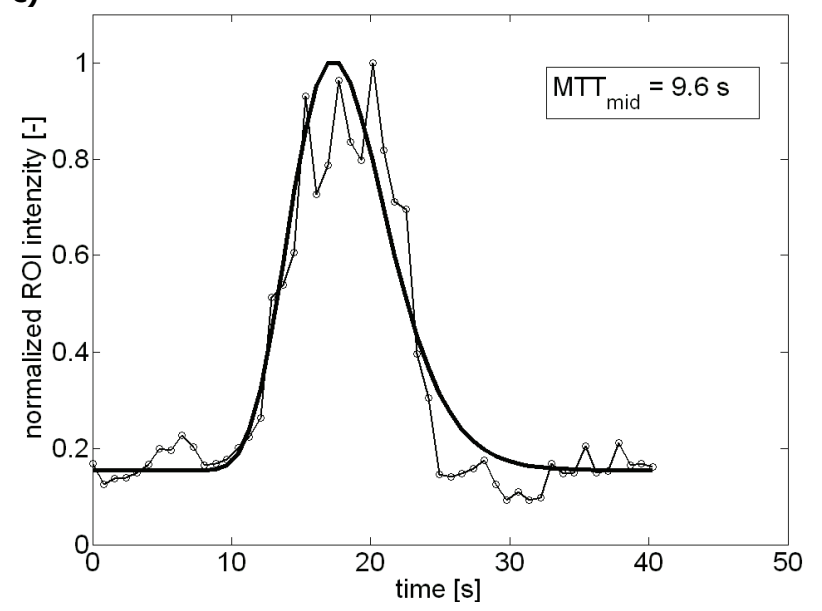

d)

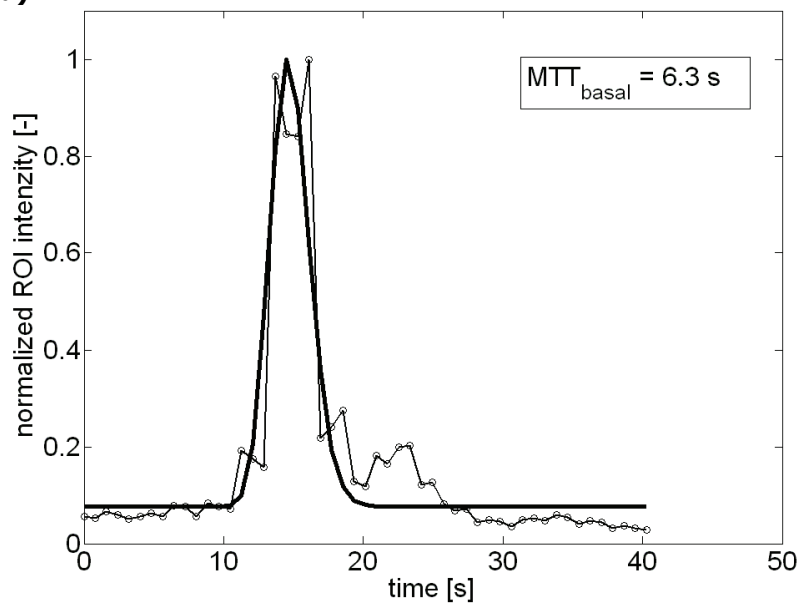

Fig. 4. a) The rough position of three analyzed myocardial regions; b, c, d) Three measured and fitted perfusion curves obtained from ROIs in the apical septum segment (b), mid septum segment (c) and basal septum segment (d). The corresponding values of the $M T T$ are shown in respective curves.

a promising tool for a less invasive assessment of myocardial perfusion in comparison to the well accepted standard represented by positron (or single photon) emission computed tomography. The main problems of fully automatic perfusion analysis in echocardiography in clinical practice are moving artifacts, ultrasound attenuation due to tissue and due to time-varying UCA concentration and also segmentation of the blood pool and myocardium. The choice of the underlying pharmacokinetic model and the parameter-estimation procedure remains an open question. Also the acquisition protocols have not been standardized yet. The choice of the acquisition parameters is based on physician's experience. This concerns mainly the UCA dosage (UCA volume and concentration) and the ultrasound scanner setting (mainly the used frequency, mechanical index and imaging mode). 

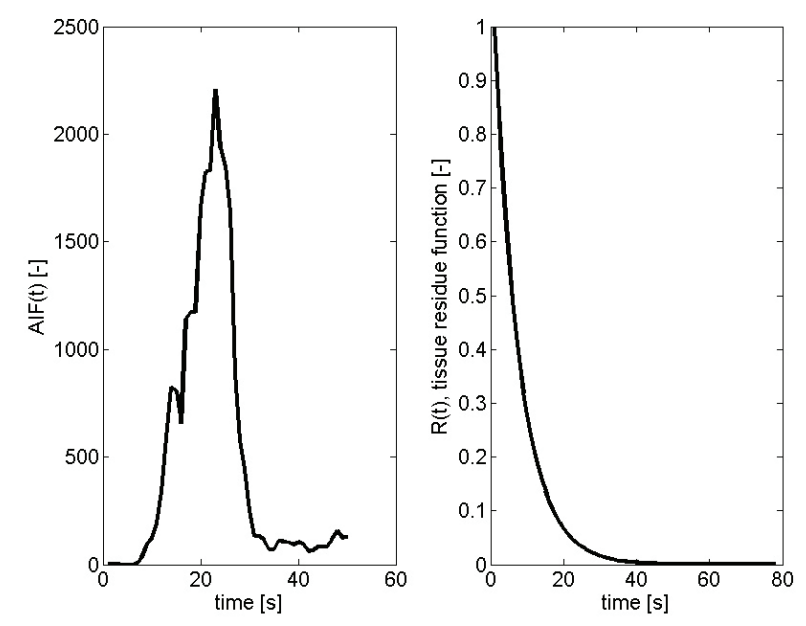

\section{Conflict of Interest}

There is no conflict of interest.

\section{Acknowledgement}

This work has been supported by the project of Czech Science Foundation no. GA $102 / 09 / 1690$ and by the institutional research frame no. MSM 0021630513

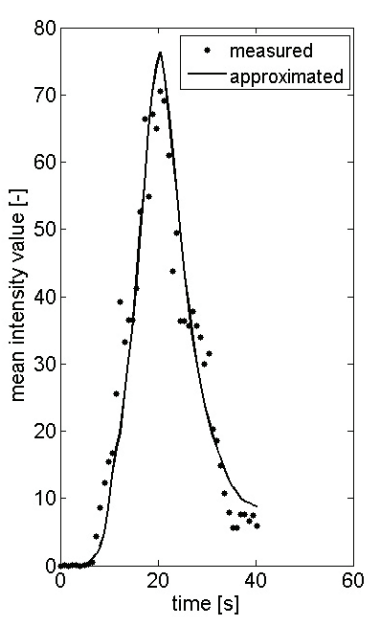

Fig. 5. Example curves used in deconvolution-based perfusion analysis. Left: $\operatorname{AIF}(t)$ measured in the left ventricle. Middle: Tissue residue function estimated by deconvolution. Right: Measured and approximated perfusion curve $c(t)$.

\section{References}

sponsored by the Ministry of Education of the Czech Republic. The authors would like to thank to doc. MUDr. Václav Chaloupka, CSc. (Department of Cardiopulmonary Testing, Faculty Hospital Brno) for his valuable comments and for providing the ultrasound image sequences.

AKINBOBOYE OO, IDRIS O, CHOU RL, SCIACCA RR, CANNON PJ, BERGMANN SR: Absolute quantitation of coronary steal induced by intravenous dipyridamole. J Am Coll Cardiol 37: 109-116, 2001.

ARDITI M, FRINKING P, ZHOU X, ROGNIN N: A new formalism for the quantification of tissue perfusion by the destruction-replenishment method in contrast ultrasound imaging. IEEE Trans Ultrason Ferroelectr Freq Control 53: 1118-1129, 2006.

BARTOŠ M, JIŘÍK R, HARABIŠ V, KOLÁř R: Measurement of SonoVue ${ }^{\mathrm{TM}}$ properties for quantitative contrast ultrasonic imaging. In: Biosignal 2008 Proceedings, Analysis of Biomedical Signals and Images. VUTIUM Press, Brno, 2008.

CHEN Q, ZAGZEBSKI J, WILSON T, STILES T: Pressure-dependent attenuation in ultrasound contrast agents. Ultrasound Med Biol 28: 1041-1051, 2002.

EYDING J, WILKENING W, POSTERT T: Brain perfusion and ultrasonic imaging techniques. Eur J Ultrasound 16: 91-104, 2002.

FORSBERG F, LIU J, SHI W, RO R, JAMES K, DENG X, HALL A: Perfusion estimation using subharmonic contrast microbubble signals. Proceedings of 2004 IEEE Ultrasonics Symposium 1: 5-8, 2004.

GARDNER EA, SUMANAWEERA TS, WOELMER MN, STEINS RW, LEEN E: Removing local motion from ultrasonic images using nonaffine registration for contrast quantification. Proceedings of 2004 IEEE Ultrasonics Symposium 3: 1718- 1721, 2004.

GREIS C: Technology overview: SonoVue. Eur Radiol Suppl 14: P11-P15, 2004.

HOFF L: Acoustic Characterization of Contrast Agents for Medical Ultrasound Imaging. Kluwer Academic Publisher, Dordrecht, 2001.

HOSSACK J, LI Y, YANG Z, FRENCH B: Assessment of transient myocardial perfusion defects in intact mice using a microbubble contrast destruction/refill approach. Proceedings of 2004 IEEE Ultrasonics Symposium 1: 9-12, 2004. 
HUNDLEY W, KIZILBASH AM, AFRIDI I, FRANCO F, PESHOCK RM, GRAYBURN PA: Administration of an intravenous perfluorocarbon contrast agent improves echocardiographic determination of left ventricular volumes and ejection fraction: comparison with cine magnetic resonance imaging. J Am Coll Cardiology 32: 1426-1432, 1998.

JACKSON A, BUCKLEY DL, PARKER M: Dynamic Contrast-Enhanced Magnetic Resonance Imaging in Oncology. Springer, Berlin, 2005.

JAN J: Digital Signal Filtering, Analysis and Restoration. The Institution of Electrical Engineers, London, 2000.

KAUL S, SENIOR R, DITTRICH H, RAVAL U, KHATTAR R, LAHIRI A: Detection of coronary artery disease with myocardial contrast echocardiography: comparison with 99mTc-sestamibi single-photon emission CT. Circulation 96: 785-792, 1997.

KOLAR R, JIRIK R, HARABIS V, NYLUND K, GILJA OH: Registration of ultrasound contrast images for perfusion analysis. Proceedings of 2009 IEEE International Ultrasonics Symposium, 2009 (in press).

KORNBLUTH M, LIANG DH, BROWN P, GESSFORD E, SCHNIGER I: Contrast echocardiography is superior to tissue harmonics for assessment of left ventricular function in mechanically ventilated patients. Am Heart J 140: 291-296, 2000.

LANG RM, MOR-AVI V: Clinical utility of contrast-enhanced echocardiography. Clin Cardiol 29: 15-25, 2006.

MALM S, FRIGSTAD S, HELLAND F, OYE K, SLORDAHL S, SKJARPE K: Quantification of resting myocardial blood flow velocity in normal humans using real-time contrast echocardiography: a feasibility study. Cardiovasc Ultrasound 3: 16-25, 2005.

MISCHI M, JANSEN A, KALKER A, KORSTEN H: Identification of ultrasound contrast agent dilution systems for ejection fraction measurements. IEEE Trans Ultrason Ferroelectr Freq Control 52: 410-420, 2005.

MISCHI M, DEN BOER JA, KORSTEN H: On the physical and stochastic representation of an indicator dilution curve as a gamma variate. Physiol Meas 29: 281-294, 2008.

MULE S, CESARE AD, LUCIDARME O, FROUIN F, HERMET A: Regularized estimation of contrast agent attenuation to improve the imaging of microbubbles in small animal studies. Ultrasound Biol 34: 938-948, 2008.

MÖHLENKAMP S, BEIGHLEY PE, PFEIFER EA, BEHRENBECK TR, SHEEDY PF, RITMAN EL: Intramyocardial blood volume, perfusion and transit time in response to embolization of different sized microvessels. Cardiovasc Res 57: 843-852, 2003.

MULVAGH SL, DE MARIA AN, FEINSTEIN SB, BURNS PN, KAUL S, MILLER JG, MONAGHAN M, PORTER TR, SHAW LJ, VILLANUEVA FS: Contrast echocardiography: current and future applications. $J$ Am Soc Echocardiogr 13: 331-342, 2000.

OPPENHEIM AV, SCHAFER RW: Discrete-Time Signal Processing. Prentice-Hall, Boston, 2009.

OSTERGAARD L: Principles of cerebral perfusion imaging by bolus tracking. J Magn Reson Imaging 22: 710-717 2005.

POTDEVIN T, FOWLKES J, MOSKALIK A, CARSON P: Reticulated foam flow phantom ultrasound contrast agent studies. Proceedings of 2002 IEEE Ultrasonics Symposium 2: 1973-1976, 2002.

QUAIA E: Contrast Media in Ultrasonography: Basic Principles and Clinical Applications. Springer-Verlag, Berlin, 2005.

RAKHIT DJ, BECHER H, MONAGHAN M, NIHOYANNOPOULIS P, SENIOR R: The clinical applications of myocardial contrast echocardiography. Eur J Echocardiogr 8: 24-29, 2007.

REKTORYS K: Survey of Applicable Mathematics. Kluwer, Dordrecht, 1994.

RIM SJ, LEONG-POI H, LINDNER JR, COUTURE D, ELLEGALA D, MASON H, DURIEX M, KASSEL NF, KAUL S: Quantification of cerebral perfusion with "real-time" contrast-enhanced ultrasound. Circulation 104: 2582-2587, 2001.

ROGNIN NG, FRINKING P, COSTA M, ARDITI M: In-vivo perfusion quantification by contrast ultrasound: validation of the use of linearized video data vs. raw RF data. Proceedings of 2008 IEEE International Ultrasonics Symposium: 1690-1693, 2008.

SHEPPARD CW, SAVAGE LJ: The random walk problem in relation to the physiology of circulatory mixing. Phys Rev 83: 489-490, 1951. 
VANNAN BK: Imaging techniques for the myocardial contrast echocardiography. Eur J Echocardiogr 1: 224-226, 2000.

WEI K, JAYAWEERA AR, FIROOZAN S, LINKA A, SKYBA DM, KAUL S: Quantification of myocardial blood flow with ultrasound-induced destruction of microbubbles administered as a constant venous infusion. Circulation 97: 473-483, 1998.

WEI K, LE E, BIN J, COGGINS M, THORPE J, KAUL S: Quantification of renal flow with contrast-enhanced ultrasound. J Am Coll Cardiol 37: 1135-1140, 2001.

ZWIRN G, BEERI R, GILON D, FRIEDMAN Z, AKSELROD S: Quantitative evaluation of local myocardial blood volume in contrast echocardiography. Med Image Anal 13: 62-79, 2009. 